\title{
Psychometric Properties of the Measure of Adolescent Coping Strategies (MACS)
}

\author{
Sigrun Sveinbjornsdottir ${ }^{1}$, Einar Baldvin Thorsteinsson ${ }^{2}$ \\ ${ }^{1}$ University of Akureyri, Akureyri, Iceland \\ ${ }^{2}$ University of New England, Armidale, Australia \\ Email: sigrunsv@unak.is, ethorste@une.edu.au
}

Received December $18^{\text {th }}$, 2013; revised January $15^{\text {th }}, 2014$; accepted February $14^{\text {th }}, 2014$

\begin{abstract}
Copyright (C 2014 Sigrun Sveinbjornsdottir, Einar Baldvin Thorsteinsson. This is an open access article distributed under the Creative Commons Attribution License, which permits unrestricted use, distribution, and reproduction in any medium, provided the original work is properly cited. In accordance of the Creative Commons Attribution License all Copyrights (C) 2014 are reserved for SCIRP and the owner of the intellectual property Sigrun Sveinbjornsdottir, Einar Baldvin Thorsteinsson. All Copyright (C) 2014 are guarded by law and by SCIRP as a guardian.
\end{abstract}

This paper presents the collective results of three independent studies in two countries, Australia and Iceland, aimed to develop a psychometrically sound adolescent coping scale, the Measure of Adolescent Coping Strategies (MACS). Study 1, relying on 3034 Australian and 6908 Icelandic adolescents, revealed five conceptually distinct first-order factors. Two second-order factors (dimensions) were derived: (a) adaptive coping encompassing stoicism/distraction, seeking social support, and self-care, and (b) maladaptive coping encompassing acting out and rumination. This factor structure was supported by multisample confirmatory factor analysis (MSCFA) across age, gender and national samples. Study 1 established evidence of discriminant validity. In Study 2, including 534 Australian adolescents, the factor structure was verified through confirmatory factor analysis (CFA). Studies 1 and 2 provided evidence of construct validity. Study 3, employing 118 Icelandic adolescents, established 16-week test-retest reliability and evidence of convergent validity. Therefore, the MACS is a short, reliable and valid scale to measure adolescent coping, thus enabling clinicians, researchers and educators to improve their practice when conducting research and guiding adolescents in developing effective coping methods.

Keywords: Adolescent; Coping; Cross-Cultural; Scale; Psychometrics

\section{Introduction}

How people cope with their daily hassles is believed to affect their health and well-being (Lazarus, 1993, 2000). The World Health Organization (WHO, 2003, 2006, 2007) has raised concerns for adolescents' mental health and psychosocial development in particular, and identified poor coping skills as one of several risk factors for ill health in adulthood and even premature death.

Lazarus and Folkman's (1984) theory of coping has inspired coping research through decades. The theory suggests that coping is two-dimensional; problem-focused, aimed at changing the situation or managing the problem causing the stress and emotion-focused, adjusting or controlling the emotions related to the problem. However, there is still confusion surrounding the concept and measurement of adults', adolescents' and children's ways of coping (e.g., Compas, Connor-Smith, Saltzman, Thomsen, \& Wadsworth, 2001; Schwarzer \& Schwarzer, 1996; Steger, 2006). Reviews of coping scales reveal inconsistent factor structure across samples (for an exhaustive review of diverse coping measures, see Compas et al., 2001; Skinner, Edge, Altman, \& Sherwood, 2003) and diverse psychometric shortcomings regarding adolescent coping scales (Sveinbjornsdottir \& Thorsteinsson, 2008). Some have argued that using factor analysis for determining lower-order factors (bottom-up) and deductive approaches for the determination of dimensions or higher-order factors (top-down; a priori decided categories) may be necessary for grasping the construct of coping (Skinner et al., 2003). Others suggest further empirical research (Sveinbjornsdottir \& Thorsteinsson, 2008).

The primary aim of the present study is to provide an empirically derived coping scale for adolescents. Hence, through three studies independent of each other, the Measurement of Adolescent Coping Strategies (MACS) will be developed. The objectives are three: 1) identify a latent structure of adolescent coping through implementation of exploratory factor analysis (EFA) on a large cross-cultural construction sample (Study 1) and a CFA on an independent sample (Study 2); 2) test for factorial invariance across age (Year 7 and Year 10), gender (female and male) and national samples (Australia and Iceland) (Study 1); and 3) test for evidence of discriminant validity (Study 1 and Study 2), convergent validity and test-retest reliability (Study 3). It was hypothesized that (a) EFA of the MACS items would reveal first-and second-order factors (i.e., dimensions), (b) the factor structure would remain invariant of adolescents' age, gender and country, and (c) factors would have acceptable evidence of convergent and discriminant validity and test-retest reliability. 


\section{Method}

\section{Samples}

Study 1 was conducted in Iceland and Australia. It was population-based in Iceland, including Year 7 and Year 10 students in primary and lower secondary schools (co-educational public schools). A total of 157 Icelandic schools participated, comprising $82 \%$ of the cohorts (7800 students). A total of 6908 students (89\%) accepted participation, with 50\% being female. In Victoria, Australia, 16 Catholic secondary schools (six single-sex girls' schools, three single-sex boys' schools, and seven co-educational schools) in inner and outer metropolitan Melbourne and rural areas participated (3782 students). A total of 3034 students (80.1\%) accepted participation, with $60 \%$ being female.

The mean age for Year 7 was 12.33 years $(S D=0.56)$ and 15.28 years $(S D=0.57)$ for Year 10 for the Australians; and 12.04 years $(S D=0.25)$ and 15.07 years $(S D=0.30)$ for the Icelanders. The Australian sample reflected a diverse ethnic background of the population with $89.9 \%$ of students, $57.3 \%$ of fathers, and $62.3 \%$ of mothers born in Australia. Others in the sample were from 82 countries and all continents. The Icelandic population-based sample reflected a homogeneous ethnic background. Born in Iceland were $95.1 \%$ of students, $95.4 \%$ of fathers and $96.7 \%$ of mothers. Others in the sample were from 60 countries in North West Europe (students $=3.6 \%$, fathers = $2.1 \%$, mothers $=1.4 \%$ ) and less than $1 \%$ from all remaining continents. Socio-economic background, as measured by parents' educational level and their professions was similar in both countries and reflected the distribution of socio-economic status in each country.

Study 2 was conducted in Australia. Participants were 534 students from five co-educational government schools and one independent single sex girls' school in inner and outer metropolitan Melbourne. The mean age was $12.55(S D=0.58)$ for Year 7 and $15.36(S D=0.53)$ for Year 10 , with $59 \%$ being female. Their ethnic background and socio-economic status was varied which is similar to the Australian participants in Study 1.

In Study 3, two schools from one Icelandic town were randomly selected and 118 students, $46 \%$ females, with a mean age of $11.76(S D=0.43)$ for Year 7 and $14.86(S D=0.35)$ for Year 10, agreed to participate. Participants' ethnic background was homogeneous, and their socio-economic status was comparable with the Icelandic participants in Study 1.

\section{Measures}

Social support. The Social Support Questionnaire Short Form (SSQ6; Sarason, Sarason, Shearin, \& Pierce, 1987) measured perceived support satisfaction (SSQS) ranging from 1 (very dissatisfied) to 6 (very satisfied) and the perceived size of the available social support network (SSQN) ranging from 0 to 9. The SSQ6 was developed using samples of older adolescents and young adults. Psychometric properties for the SSQ short version are rated as similar to the full 27-item version. Testretest reliability for the full 27-item version of SSQN and SSQS (four-week interval) is .83 and .90, respectively, and internal consistency (Cronbach's alpha) is .94 and .97, respectively (Sarason, Levine, Basham, \& Sarason, 1983). The SSQ6 has been used in studies of adolescents 14 and 16 years old (Dumont \& Provost, 1999) with reported internal consistency above .90 for both scales, and in studies of children and younger adolescents from 11 to 12 years, with internal consistency of .91 for SSQN and .86 for SSQS (Johnson, EspositoSmythers, Miranda, Rizzo, Justus, \& Clum, 2010) and .73 for SSQN (Bal, Crombez, Van Oost, \& Debourdeaudhuij, 2003). In Study 1, the internal consistency for SSQS was .90 and for SSQN .91.

Self-Esteem. Rosenberg's Global Self-Esteem Scale (RSE; Rosenberg, 1965) consisting of 10 items as adapted by Bachman (1978) was employed. Students tick one of five boxes for each item (ranging from "almost always true" to "never true"). Six items are positively worded (e.g., "When I do a job, I do it well”), and four negatively (e.g., "I feel I can't do anything right"). Items were scored from 1 to 5 with higher scores indicating higher self-esteem. Internal consistency is .93; test-retest reliability (two-week interval) of .85; and evidence of validity as indicated through correlations of the RSE with other measures of self-esteem from .56 to .83 (Chiu, 1988; Hagborg, 1993). The internal consistency was .82 in Study 1.

Coping. The KIDCOPE is a 10-item adolescent coping checklist (Spirito, Stark, \& Williams, 1988). The KIDCOPE items were selected from commonly mentioned strategies or factors in the adult coping literature and represent: problem solving, distraction, social support, social withdrawal, cognitive restructuring, self-criticism, blaming others, emotional regulation, wishful thinking, and resignation. For the purpose of Study 3, the item of emotional regulation was divided into two items, one positive and the other negative, and the modified KIDCOPE thus contained 11 items. Responses were rated on a 4-point Likert scale from 0 (I did not use) to 3 (I used almost all the time). Test-retest correlations are reported as moderate for a three-day interval (.55 to $.75, M=.65)$, lower with a sevenday interval (.07 to $.83, M=.50)$, and lowest with a 10 -week interval (.17 to $.43, M=.27$ ), and validation studies indicate moderate correlations ranging from .33 to .77 for KIDCOPE items with the Coping Strategies Inventory (Spirito et al., 1988; Tobin, Holroyd, Reynolds, \& Wigal, 1989). Internal consistency cannot be assessed for the modified KIDCOPE as it is a checklist and does not comprise factors.

\section{Procedure}

Permission for each of the three studies was gained from local and national authorities, and their policies for parental consent were followed. For Studies 1 and 3, permission was thus given via parents' lack of objection (passive consent). For Study 2, parental written consent was received for participating students (active consent).

Studies 1 and 2 were anonymous. Since test-retest purposes were incorporated in Study 3, confidentiality was ensured such that each participant had a unique code allocated. This code appeared on the questionnaire and in a separate key associating code with name. The key was kept separate (physically and by another person) from the questionnaires. After 16 weeks, the participants were again given questionnaires with their respective codes by the research assistant who had the key. The key was then destroyed.

Questionnaires were administered during school hours with an average of 25 students per administration. Standard written information was read to participants: participants were to think of a stressor and rate their responses to the MACS items based upon this stressor. In Study 3, students used the same stressor for both administrations. 
The questionnaire package was developed in English and translated into Icelandic through a combination of back-translation and committee approach (Brislin, Lonner, \& Thorndike, 1973).

\section{Item Selection}

The original idea of developing an adolescent coping scale, on which the MACS is based, stemmed from late Dr Chris Madden's interest in athletes coping with competitive stress (Madden, 1987; Madden, Kirkby, \& McDonald, 1989; Madden, Summers, \& Brown, 1990) and his clinical experience working with drug addicts (Madden, 1989). Originally, Madden accommodated Lazarus' theory on emotion-focused versus problem-focused coping (Lazarus \& Folkman, 1984) and developed the Ways of Coping with Sport (WOCS; Madden et al., 1989), a 64-item scale that was adapted from the Ways of Coping Checklist (WOCC; Folkman \& Lazarus, 1985) to suit competitive athletes. Under the direction of Dr Madden, a group at La Trobe University in Melbourne also developed an adolescent coping scale for general concerns, the Coping Questionnaire for Adolescents (CQA; Angleton, 1994). The CQA included 34 items drawn from the coping literature and the WOCS. The wording of items was adapted for use with adolescents. The CQA also included an open-ended item asking about additional coping strategies. The CQA was administered to 261 Year 10 students in Melbourne (mean age 15.03 years, $S D=0.60,54 \%$ males) with varied socio-economic and ethnic backgrounds (Angleton, 1994). The students identified their own stressor experienced during the last twelve months and completed the CQA according to that stressor. Their self-generated coping strategies were classified and a total of 19 items were created on the basis of frequency.

There were 53 pre-MACS items on which the final version of the MACS was based, including the 19 items generated from the adolescents and the 34 items of the CQA.

\section{Scale Development}

In Study 1, the following choices of EFA procedures were employed: (a) common factor analysis (e.g., principal axis factoring) (e.g., Fabrigar, Wegener, MacCallum, \& Strahn, 1999; Gorsuch, 1983; Widaman, 1993); (b) oblique rotation (e.g., Oblimin) (Child, 1990; Kline, 1994); and (c) parallel analysis (PA), Velicer's minimum average partial (MAP) and scree plots. Final item selection from the pool of 53 based on: (a) a full range distribution of responses for each item on the four response scores; (b) each factor include a minimum of five items (Gorsuch, 1983); (c) each item load beyond .25 on one factor only (Fabrigar et al., 1999; Widaman, 1993); (d) each factor's internal consistency being at least .70; (e) mean inter-item correlations $\left(M_{\mathrm{r}}\right.$ ) for each factor being between .20 and .40 (Briggs \& Cheek, 1986); (f) face validity; and (g) conceptually distinct factors.

In Study 2, a CFA of the MACS final factor structure at firstand second-order level was tested using AMOS structural equation modeling software (Byrne, 2010; Kline, 2011). Missing values were imputed using covariance-based statistics or the expectation-maximization (EM) method (normal distribution assumption). Structural equation model indices were selected to cover the main facets of model fit. The Goodness-of-Fit Index (GFI) was used as an absolute fix index; GFI ranges from 0 to 1 , values above .95 indicating a good fit. Comparative Fit Index (CFI) was calculated. It ranges from 0 to 1 and values above 90 suggest a good fit. The root mean square error of approximation (RMSEA) and squared root mean residual (SRMR) were calculated. SRMR with a value of 0 represents a perfect fit between the model and the population covariance matrix. RMSEA values below .06 and SRMR values below.09 are generally interpreted as favourable. Finally, the Tucker-Lewis coefficient (TLI) was reported, values close to 1.0 indicating good fit.

A multi-sample CFA (MSCFA; Kline, 2011) was conducted for the second-order factor model using the data from Study 1. Factorial invariance was examined for gender (male, female), age (Year 7, Year 10), and country (Australia, Iceland). A criteria of .01 change in CFI $(\triangle \mathrm{CFI})$ from the configural model (unrestrained) to the measurement model (restrained) was used to determine if invariance should be rejected or not.

In Studies 1 and 2, the SSQ6 (i.e., SSQS and SSQN) and the RSE as adapted by Bachman, O’ (1978) were employed to provide evidence of discriminant validity. It was hypothesized that correlations between MACS, SSQ6 and RSE would be low.

Finally, in Study 3, the KIDCOPE (Spirito et al., 1988) was chosen to establish evidence of convergent validity of the MACS. Moderate correlations between a presumably psychometrically sound test and a poor(er) one is expected (Kline, 1986). It was hypothesized that the first-order factors of MACS would correlate moderately with the KIDCOPE.

\section{Results}

\section{Factor Analysis: First Order}

Principal axis factoring (PAF) with Oblimin rotation was performed on the data from Study 1. The data was then divided into four sub-samples (Australia Year 7 and Year 10; Iceland Year 7 and Year 10) and factor analyses performed on all groups. Items with low loadings for all groups or loading significantly on more than one factor were deleted. Final factor structure was derived after stepwise reduction of items. Five first-order factors of coping were detected in Study $1(N=9942)$ based on 34 of the original 53 items. Thirty items loaded $\geq .25$ on one factor only. Four items loaded on two factors; these items were included in their higher loading factor. The number of factors was based on a Scree plot suggesting five factors, a parallel analysis suggesting six factors, and MAP test suggesting four factors. Comparable factor structures emerged for both age-groups in Iceland (Year 7 and 10) and for the older group in Australia (Year 10) (stoicism/distraction, acting out, rumination, seeking social support, and self-care). For the fourth subsample (Australian Year 7), the 34 items comprised four factors with items from the stoicism/distraction and self-care factors combined into one factor. Table 1 provides information on internal consistency, mean inter-item correlations in factors, and number of items per factor. A CFA, using data from Study 2, supported the factor structure with CFI $=.95$, TLI $=.94$, GFI $=.96$, RMSEA $=.047$ [90\% CI .038, .057], and SRMR $=.046$.

\section{Factor Analysis: Second Order}

Second-order factor analysis (PAF, Oblimin rotation) revealed two dimensions of coping. One was adaptive (i.e., stoicism/distraction, self-care, and seeking social support) and the 
Table 1.

The five coping factors of the MACS, coefficient Alpha and mean inter-item correlation (Mr) for each factor for study 1, N = 9942.

\begin{tabular}{cccc}
\hline Factor & Alpha & $M_{r}$ & Number of items \\
\hline Stoicism/distraction & .73 & .25 & 8 \\
Acting out & .75 & .34 & 6 \\
Rumination & .70 & .28 & 6 \\
Seeking social support & .81 & .37 & 7 \\
Self-care & .70 & .25 & 7 \\
\hline
\end{tabular}

other maladaptive (i.e., rumination and acting out; see Table 2). A CFA for this model, including first- and second-order factors using data from Study 2 was supported, CFI $=.94$, TLI $=.92$, GFI = .95, RMSEA $=.054$ [90\% CI .045, .064], and SRMR $=.063$.

\section{Factor Invariance}

MSCFA was used to investigate factorial invariance in Study 1 for country, gender, and year at school. It was supported for all three demographic factors examined; $\Delta \mathrm{CFI}<.01$ (see Table 3).

\section{Convergent Validity}

Evidence of convergent validity was assessed in Study 3 by examining the correlations between the first-order factors of the MACS and the items of the modified KIDCOPE. As hypothesized, there were moderate correlations between the 11 items of the modified KIDCOPE and the corresponding five first-order factors of MACS, with $\mathrm{r}$ ranging from .25 to .68 and with a mean around .46 .

\section{Discriminant Validity}

In Studies 1 and 2, evidence of discriminant validity was assessed for the five first-order coping factors of the MACS based on correlations with SSQN, SSQS, and RSE. Low correlations were expected and found between SSQN and all factors of MACS , ranging from $r=-.05$ to $r=.11$ in Study 1 and Study 2. The correlations between SSQS and the factors of MACS were also low, ranging from $r=.02$ to $r=.17$. The correlation between RSE and the factors of MACS was somewhat higher but within expected range, ranging from $r=-.27$ to $r=.12$.

\section{Reliability}

A test-retest reliability of .50 was expected for a 16 -week interval, given the age and plausible development of participants during the period. Study 3 found test-retest reliability for a 16-week interval as follows: seeking social support $r=.74$; self-care $r=.68$; acting out $r=.59$; rumination $r=.74$; and stoicism/distraction $r=.53$; all with $p<.001$.

\section{Discussion}

The primary aim of the present studies was achieved, that is to establish through empirical research a psychometricallyderived measure of adolescent coping strategies, the MACS. All three hypothesis were supported: (a) EFA of the MACS items revealed first- and second-order factors (i.e. dimensions),
Table 2.

Pattern matrix for two second-order factors using principal axis factoring with oblimin rotation for study $1, \mathrm{~N}=9942$.

\begin{tabular}{ccc}
\hline Factor & Adaptive coping & Maladaptive coping \\
\hline Stoicism/distraction & .78 & -.09 \\
Self-care & .65 & -.03 \\
Seeking social support & .61 & .17 \\
Rumination & .15 & .78 \\
Acting out & -.04 & .33 \\
\hline
\end{tabular}

Note: The cut-off limit for first-order factor loadings on second-order factors is .25 .

(b) the factor structure remained invariant of adolescents' age, gender and country, and (c) evidence of convergent and discriminant validity, and test-retest reliability was acceptable.

The five first-order factors of the MACS, comprising six to eight items each, were conceptually distinct and labeled in relation to the main content of the strategies included. All factors included both cognitive and behavioral strategies, and either problem-solving or avoidant strategies.

The first factor, stoicism/distraction, captures the way individuals manage stressful events or situations without trying to solve the problem or directly change the situation for the better. The adolescents are aware of the stressful situation but try not to let it affect them, such as distracting themselves by watching TV or through play. These are possibly the only positive coping strategies if situations are uncontrollable (e.g., awaiting surgery).

The second factor, acting out, refers to destructive behavior towards other people or things, and towards oneself, including aggressive behavior, use of alcohol and drugs, and yelling rather than discussing. This coping factor was the least employed by both females and males. Similar to stoicism/distraction, acting out does not aim at solving a stressful situation. However, it includes antisocial and self-destructive behavior which may seem helpful in the short term but are likely to turn maladaptive in the long run. Antisocial behavior at the age of 11 may increase the risk of such behavior later (White, Moffitt, Earls, Robins, \& Silva, 1990) and the same applies for substance use (Thorlindsson \& Vilhjalmsson, 1991).

The third factor, rumination, includes negative feelings and thoughts towards oneself, dwelling on the problem, and wishful thinking, and does not solve the stressful situation. This coping factor was the only one where gender differences were noted, females employing it more than males. That supports previous literature on rumination (e.g., Nolen-Hoeksema, Wisco, \& Lyubomirsky, 2008).

The fourth coping factor, seeking social support, deals with the stressful situation. It refers to active problem-focused strategies, mainly cognitive, such as talking to other people, seeking advice and getting ideas, or just sharing the problem with someone to ease the burden. This is the most common way of coping for both females and males; it aims to strengthen the individual as well as to solve a stressful situation.

The fifth and last factor, self-care, includes physical and psychological care. The adolescents exercise, sleep and eat well, pray and look after themselves. The stressful situation is not solved or the problem directly addressed. Adolescents seek to strengthen their body and spirit to help them withstand difficulties (e.g., upcoming exams, parental illness). 
Table 3.

Multi-sample confirmatory factor analysis examining factorial invariance for different demographics in study 1 second-order factor model.

\begin{tabular}{|c|c|c|c|c|c|c|}
\hline Factorial invariance & CFI & TLI & GFI & RMSEA & $90 \%$ CI & SRMR \\
\hline Comparing males vs. females & .929 & .907 & .957 & .045 & $.043, .046$ & $<.05$ \\
\hline Configural model (unrestrained) & .929 & .907 & .957 & .045 & $.043, .046$ & $<.05$ \\
\hline Measurement model (restrained) & .923 & .909 & .953 & .043 & $.043, .045$ & $<.06$ \\
\hline$\Delta \mathrm{CFI}$ & .006 & & & & & \\
\hline \multicolumn{7}{|l|}{ Comparing Year 7 vs. Year 10} \\
\hline Unrestrained & .925 & .902 & .955 & .046 & $.045, .048$ & $<.05$ \\
\hline Restrained & .921 & .907 & .951 & .045 & $.044, .046$ & $<.06$ \\
\hline$\Delta \mathrm{CFI}$ & .004 & & & & & \\
\hline \multicolumn{7}{|l|}{ Comparing Australia vs. Iceland } \\
\hline Unrestrained & .924 & .901 & .954 & .046 & $.045, .048$ & $<.06$ \\
\hline Restrained & .920 & .906 & .951 & .045 & $.044, .046$ & $<.07$ \\
\hline$\Delta \mathrm{CFI}$ & .004 & & & & & \\
\hline
\end{tabular}

Note: CFI = Comparative Fit Index; TLI = Tucker-Lewis Coefficient; GFI = Goodness-of-Fit Index; RMSEA = Root Mean Square Error of Approximation; SRMR = Squared Root Mean Residual; $\triangle \mathrm{CFI}=$ Change in CFI from unconstrained to constrained.

A second-order factor analysis clearly suggests that adolescent coping is two-dimensional. One dimension includes the first-order factors seeking social support, self-care and stoicism/distraction; and the other, rumination and acting out. Given the content of the two second-order factors, and the possible consequences each implies for adolescents, the former may be recognized as adaptive and the latter maladaptive. Therefore, it is suggested that in relation to health and well-being, the two second-order factors refer to: (a) adaptive coping as an indication of protection; and (b) maladaptive coping as an indication of risk.

The two empirically derived second-order factors of the MACS (adaptive versus maladaptive coping) both comprise behaviorally-and cognitively-based strategies that are either active or inactive. Adaptive coping includes both problem-and emotion-focused coping (i.e., aiming at changing the situation/ managing the problem; and adjusting or controlling the emotions related to the problem) and it also includes either approaching the problem or avoiding it. In contrast, maladaptive coping is emotion-focused and not problem-focused: the problem is avoided and not approached.

The present study is limited in its reliance on factor analysis which always has a subjective component to it (e.g., final decision on the number of factors to extract). The MACS was developed through participation from two economically advanced populations and its validity for alternative cultures is unknown. Furthermore, it is for future research to examine the validity of the interpretations of the MACS's coping strategies as buffers to the effects of stressors on adolescents' psychological wellbeing and physical health. Such research is taking place and three projects have already been published utilizing the MACS. First, a project in Botswana found that seeking social support and distraction reduced the impact of negative life events on psychological health (Thorsteinsson, Sveinbjornsdottir, Dintsi, \& Rooke, 2013). Second, an Australian project found that 66\% of the variation in depression was explained by a mediation model where rumination, acting out and social support acted as partial mediators of the perceived stress-depression relationship (Thorsteinsson, Ryan, \& Sveinbjornsdottir, 2013). Third, an Australian longitudinal study suggests that self care and rumination may affect change in compulsive Internet use and depression, respectively (Thorsteinsson \& Davey, 2014).

\section{Conclusion}

Researchers and practitioners (e.g., psychologists and educators) should find the MACS a reliable and valid measure of adolescent coping that is applicable across different cultures. The MACS enables researchers and practitioners to improve their practice when conducting research and guiding adolescents in developing effective coping methods.

\section{Acknowledgements}

We would like to acknowledge the late Dr Chris C. Madden's valuable contribution to the early development of the MACS. We also thank Ms Alison Angleton for her contribution to the item selection of MACS.

This research was supported by a grant from The Icelandic Centre for Research (RANNIS).

\section{REFERENCES}

Angleton, A. (1994). Specific and transitional stress, self-efficacy, and coping of students in three different stages of adolescent development. Melbourne: La Trobe University.

Bachman, J. G., O’Malley, P. M., \& Johnston, J. (1978). Self-esteem and attainment: An analysis of change and stability. In J. G. Bachman, P. M. O’Malley, \& J. Johnston (Eds.), Youth in transition. Adolescence to adulthood-Change and stability in the lives of young men (pp. 93-120). Ann Arbor, Michigan: Institute for Social Research, The University of Michigan.

Bal, S., Crombez, G., Van Oost, P., \& Debourdeaudhuij, I. (2003). The role of social support in well being and coping with self reported stressful events in adolescents. Child Abuse \& Neglect, 27, 13771395. http://dx.doi.org/10.1016/j.chiabu.2003.06.002

Beshart, M. A. (2010). Psychometric properties of short form selfregulation inventory in a sample of Iranian population. Research in Clinical Psychology and Counseling, 1, 53-70.

Briggs, S. R., \& Cheek, J. M. (1986). The role of factor analysis in the 
development and evaluation of personality scales. Journal of Personality, 54, 106-148.

http://dx.doi.org/10.1111/j.1467-6494.1986.tb00391.x

Brislin, R. W., Lonner, W. J., \& Thorndike, R. M. (1973). Cross-cultural research methods. New York: John Wiley \& Sons.

Byrne, B. M. (2010). Structural equation modelling with AMOS: Basic concpets, applications, and programming (2nd ed.). New York: Routledge.

Child, D. (1990). The essentials of factor analysis (2nd ed.). London: Cassell Educational Ltd.

Chiu, L. H. (1988). Measures of self-esteem for school-age children. Journal of Counseling and Development, 66, 298-301. http://dx.doi.org/10.1002/j.1556-6676.1988.tb00874.x

Compas, B. E., Connor-Smith, J. K., Saltzman, H., Thomsen, A. H., \& Wadsworth, M. E. (2001). Coping with stress during childhood and adolescence: Problems, progress, and potential in theory and research. Psychological Bulletin, 127, 87-127. http://dx.doi.org/10.1037/0033-2909.127.1.87

Dumont, M., \& Provost, M. A. (1999). Resilience in adolescents: Protective role of social support, coping strategies, self esteem, and social activities on experience of stress and depression. Journal of Youth and Adolescence, 28, 343-363. http://dx.doi.org/10.1023/A:1021637011732

Fabrigar, L., Wegener, D., MacCallum, R., \& Strahn, E. (1999). Evaluating the use of exploratory factor analysis in psychological research. Psychological Methods, 4, 272-299. http://dx.doi.org/10.1037/1082-989X.4.3.272

Folkman, S., \& Lazarus, R. S. (1985). If it changes it must be a process: Study of emotion and coping during three stages of a college examination. Journal of Personality and Social Psychology, 48, 150-170. http://dx.doi.org/10.1037/0022-3514.48.1.150

Gorsuch, R. (1983). Factor analysis (2nd ed.). Hillsdale, NJ: Lawrence Erlbaum Associates.

Hagborg, W. J. (1993). The Rosenberg self-esteem scale and Harter's self-perception for adolescents: A concurrent validity study. Psychology in the Schools, 30, 132-136.

http://dx.doi.org/10.1002/1520-6807(199304)30:2<132::AID-PITS23 $10300205>3.0 . \mathrm{CO} ; 2-\mathrm{Z}$

Johnson, J. E., Esposito-Smythers, C., Miranda Jr., R., Rizzo, C. J., Justus, A. N., \& Clum, G. (2010). Gender, social support, and depression in criminal justice-Involved adolescents. International Journal of Offender Therapy and Comparative Criminology, 55, 1096-1109. http://dx.doi.org/10.1177/0306624X10382637

Kline, P. (1986). A handbook of test construction: Introduction to psychometric design. London: Methuen.

Kline, P. (1994). An easy guide to factor analysis. London: Routledge.

Kline, R. B. (2011). Principles and practice of structural equation modeling (3rd ed.). New York: Guilford Press.

Lazarus, R. S. (1993). Coping theory and research: Past, present, and future. Psychosomatic Medicine, 55, 234-247.

Lazarus, R. S. (2000). Toward better research on stress and coping. American Psychologist, 55, 665-673. http://dx.doi.org/10.1037/0003-066X.55.6.665

Lazarus, R. S., \& Folkman, S. (1984). Stress, appraisal and coping. New York: Springer Publishing Company.

Madden, C. C. (1987). Coping with competitive sport. Master of Science Thesis, Melbourne: University of Melbourne.

Madden, C. C. (1989). Anxiety, depression, and coping in alcohol and drug-dependent persons undergoing rehabilitation. In R. Godding, D. Rankin, \& G. Whelan (Eds.), Proceedings of the autumn school of studies on alcohol and drugs (pp. 97-101). Melbourne: Department of Community Medicine, St. Vincent's Hospital.

Madden, C. C., Kirkby, R. J., \& McDonald, D. (1989). Coping styles of competitive middle distance runners. International Journal of Sport Psychology, 20, 287-296.

Madden, C. C., Summers, J. J., \& Brown, D. F. (1990). The influence of perceived stress on coping with competitive basketball. International Journal of Sport Psychology, 21, 21-35.

Nolen-Hoeksema, S., Wisco, B. E., \& Lyubomirsky, S. (2008). Rethinking rumination. Perspectives on Psychological Science, 3, 400-
424. http://dx.doi.org/10.1111/j.1745-6924.2008.00088.x

Rosenberg, M. (1965). Society and the adolescent self-image. Princeton, NJ: Princeton University Press.

Sarason, I. G., Levine, H. M., Basham, R. B., \& Sarason, B. R. (1983). Assessing social support: The social support questionnaire. Journal of Personality and Social Psychology, 44, 127-139. http://dx.doi.org/10.1037/0022-3514.44.1.127

Sarason, I. G., Sarason, B. R., Shearin, E. N., \& Pierce, G. R. (1987). A brief measure of social support: Practical and theoretical implications. Journal of Social and Personal Relationships, 4, 497-510.

http://dx.doi.org/10.1177/0265407587044007

Schwarzer, R., \& Schwarzer, C. (1996). A critical survey of coping instruments. In M. Zeidner, \& N. S. Endler (Eds.), Handbook of coping: Theory, research, applications (pp. 107-132). New York: John Wiley and Sons.

Skinner, E. A., Edge, K., Altman, J., \& Sherwood, H. (2003). Searching for the structure of coping: A review and critique of category systems for classifying ways of coping. Psychological Bulletin, 129, 216-269. http://dx.doi.org/10.1037/0033-2909.129.2.216

Spirito, A., Stark, L. J., \& Williams, C. (1988). Development of a brief coping checklist for use with pediatric populations. Journal of Pediatric Psychology, 13, 555-574. http://dx.doi.org/10.1093/jpepsy/13.4.555

Steger, M. F. (2006). An illustration of issues in factor extraction and identification of dimensionality in psychological assessment data. Journal of Personality Assessment, 86, 263-272. http://dx.doi.org/10.1207/s15327752jpa8603_03

Sveinbjornsdottir, S., \& Thorsteinsson, E. B. (2008). Adolescent coping scales: A critical psychometric review. Scandinavian Journal of Psychology, 49, 533-548.

http://dx.doi.org/10.1111/j.1467-9450.2008.00669.x

Thorlindsson, T., \& Vilhjalmsson, R. (1991). Factors related to cigarette smoking and alcohol use among adolescents. Adolescence, 26, 399-418.

Thorsteinsson, E. B., \& Davey, L. (2014). Adolescents' compulsive Internet use and depression: A longitudinal study. Open Journal of Depression, 3, 13-17. http://dx.doi.org/10.4236/ojd.2014.31005

Thorsteinsson, E. B., Ryan, S., \& Sveinbjornsdottir, S. (2013). The mediating effects of social support and coping on the stress-depression relationship in rural and urban adolescents. Open Journal of Depression, 2, 1-6. http://dx.doi.org/10.4236/ojd.2013.21001

Thorsteinsson, E. B., Sveinbjornsdottir, S., Dintsi, M., \& Rooke, S. E. (2013). Negative life events, distress, and coping among adolescents in Botswana. Australian Journal of Educational \& Developmental Psychology, 13, 75-86.

Tobin, D. L., Holroyd, K. A., Reynolds, R. V., \& Wigal, J. K. (1989). The hierarchical factor structure of the coping strategies inventory. Cognitive Therapy and Research, 13, 343-361. http://dx.doi.org/10.1007/BF01173478

White, J. L., Moffitt, T. E., Earls, F., Robins, L., \& Silva, P. A. (1990). How early can we tell? Predictors of childhood conduct disorder and adolescent delinquency. Criminology, 28, 507-533. http://dx.doi.org/10.1111/j.1745-9125.1990.tb01337.x

WHO (2003). Strategic directions for improving the health and development of children and adolescents. http://www.who.int/child-adolescent-health/New_Publications/Overv iew/CAH_Strategy/CAH_strategy_EN.pdf

WHO (2006). Prevention and care of illness. http://www.who.int/child-adolescent-health/PREVENTION/Adolesc ents.htm

WHO (2007). Adolescents, social support and help-seeking behaviour: An international literature review and programme consultation with recommendations for action.

http://www.who.int/maternal_child_adolescent/documents/97892415 95711/en/index.html

Widaman, K. F. (1993). Common factor analysis versus principal component analysis: Differential bias in representing model parameters? Multivariate Behavioural Research, 28, 263-311. http://dx.doi.org/10.1207/s15327906mbr2803 1 